\title{
LOS LÍMITES DEL GÉNERO LITERARIO Y EL DISCURSO CRÍTICO: EL CASO DE LA NOVELA EN MARRUECOS
}

\author{
Francisco Rodríguez Sierra \\ Universidad de Cádiz
}

\begin{abstract}
This paper copes with the distinction between literary genres properly called, and channels -or radicals- of presentation, and also with the consideration of the former ones as historically variable cultural institutions and the later ones as much more stable means of uttering. It also deals with the role of the metaliterary thought upon delimitation between genres. Thus, this paper studies the Moroccan novel case and the oscillations occurred on its generic delimitation, due to parallel oscillations happened in Moroccan critic activities.
\end{abstract}

\section{EL GÉNERO LITERARIO COMO INSTITUCIÓN SOCIAL}

Desde los años cuarenta del siglo pasado se halla extendida la idea del carácter "institucional" de la literatura. Si bien René Wellek y Austin Warren popularizaron esta idea en el capítulo "Literatura y sociedad" de su Teoría Literaria (René Wellek \& Austin Warren, 1966) ${ }^{1}$-con la que deseaban resaltar el hecho de que los procedimientos artísticos utilizados en literatura responden en esencia a convenciones nacidas y desarrolladas en el seno de la sociedad-, se inspiraron de hecho en los trabajos inmediatamente anteriores de Pearson ${ }^{2}$ y de Harry Levin ${ }^{3}$.

Estamos hablando, pues, de entidades moldeadas en el seno de la sociedad y, como tales, carentes de un ser estable universal que trascienda las épocas y las culturas. El hecho de que determinados géneros muestren una pasmosa capacidad para perpetuarse no invalida este aserto, y hay que buscar la razón precisamente en elementos de convención social y de cadencias culturales ${ }^{4}$.

\footnotetext{
1 "La literatura es una institución social que utiliza como medio propio el lenguaje, la creación social" (WellekWarren 1966: 112).

${ }^{2}$ Los géneros literarios "pueden considerarse como imperativos institucionales que se imponen al escritor y, a su vez, son impuestos por éste", citado por Wellek \& Warren 1966: 271. La cita es de Pearson: "Literary Forms and Types", English Institute Annual, 1940 (1941) 59 y ss.

3 "Cabe trabajar, expresarse a través de instituciones existentes, crear otras nuevas o seguir adelante en la medida de lo posible sin compartir políticas y rituales; cabe también adherirse a instituciones para luego reformarlas", citado por Wellek \& Warren (1966: 272), quienes se inspiran para esta frase en Harry Levin: "Literature as an Institution", Accent, VI (1946) 159-168.

${ }^{4}$ Dice Genette (1986: 146) sobre esta cuestión: "la longevidad de las formas clásicas (epopeya, tragedia) no es un índice seguro de transhistoricidad, pues en este caso se debe tener en cuenta el conservadurismo de la tradición clásica, capaz de mantener en pie durante siglos formas momificadas. Frente a tales casos de estabilidad, las formas postclásicas (o paraclásicas) padecen un desgaste histórico que se debe menos a un rasgo propio que al
} 
Esta concepción de la literatura y de los géneros literarios como institución social supone introducir en la cuestión un fuerte componente pragmático y confirmar la superación de derivaciones "genetistas" de la consideración de los géneros a favor de una inclinación más bien cultural y convencional; y supone, también, traer a colación cuestiones como el "horizonte de expectativas" de Jauss:

"Si se sustituye el concepto substancialita de género (en tanto que idea que se muestra en cada
individuo y que no se repite en tanto que género) por el concepto histórico de continuidad
(...), la relación de un texto individual con la serie de textos que constituyen el género surge
como un proceso de creación y de modificación continua de un horizonte. El nuevo texto
evoca para el lector (el oyente) el horizonte de una expectativa y de unas reglas que conoce
gracias a los textos precedentes, que sufren asimismo variaciones, rectificaciones y
modificaciones, o que son, sencillamente, reproducidos. La variación y la rectificación
delimitan el campo; la modificación y la reproducción definen los límites de la estructura del
género".

De alguna manera estamos estableciendo así una relación lógica entre el hecho, de una parte, de casar la evidencia transhistórica de unos modos, entendidos como cauces de presentación, de acuerdo a la terminología de Guillén ${ }^{6}$, con la no menos evidente presencia de las diferentes formas históricas cambiantes y sus avatares, por medio de la aceptación de estas formas cambiantes como los géneros propiamente dichos y de los cauces de presentación como instancias que atañen más a la Poética que a la teoría de los géneros ${ }^{7}$ en la medida en que no son tales géneros ${ }^{8} ; \mathrm{y}$, de otra parte, la asunción del concepto de géneros literarios como instituciones culturales. Es decir: el carácter histórico y cambiante de los géneros y su realidad institucional y cultural son facetas del mismo fenómeno. Todo ello, sumado al horizonte de expectativas, convierte el género en "punto de encuentro del acuerdo comunicativo" y "antes como un rasgo del sistema general literario, que como un conjunto de reglas performativas con incidencia en la génesis textual de la obra",

\footnotetext{
ritmo histórico. Un criterio más significativo sería la capacidad de dispersión (en diferentes culturas) y de recurrencia espontánea ( $\sin$ la ayuda de una tradición, de un revival o de una moda "retro")."

${ }^{5}$ Jauss 1986: 48-49. Este artículo de Jauss apareció originalmente en alemán en el marco de una obra colectiva: Grundriss der romanischen Literaturen des Mittelalters, Heidelberg, 1970.

${ }^{6}$ Guillén 1985: 163 y ss. Claudio Guillén habla de: 1) cauces de presentación (narración [épica], actuación [dramática] y enunciación [lírica]), 2) géneros propiamente dichos (tragedia, epopeya, ensayo); 3) modalidades, de carácter "adjetivo, parcial"; y 4) formas o "procedimientos tradicionales de interrelación, ordenación o limitación de la escritura"; donde la primera categoría se corresponde con los radicals of presentation de Northrop Frye. El concepto de cauce de presentación de Guillén equivale al de modo de enunciación de Genette (1986).

${ }^{7}$ Todorov 1988: 37: “...o bien lo lírico, lo épico, etc., son categorías universales, y por tanto del discurso (lo que no excluye que sean complejas; por ejemplo, semánticas, pragmáticas, verbales, al mismo tiempo); pero entonces pertenecen a la poética general, y no (específicamente) a la teoría de los géneros: caracterizan las manifestaciones posibles del discurso, y no las manifestaciones reales de los discursos. O bien es en los fenómenos históricos en lo que se piensa al emplear tales términos".

${ }^{8}$ Genette 1986: 142: "La diferencia de estatus entre géneros y modos está ahí en esencia: los géneros son categorías propiamente literarias ; los modos son categorías que competen a la lingüística , o más exactamente, a lo que hoy día se denomina pragmatica".

${ }^{9}$ García Berrio 1994: 603.
} 


\section{LA FORMACIÓN RETROSPECTIVA DEL GÉNERO}

La consideración, pues, de la distinción entre cauce de presentación y de género literario propiamente dicho, y, además, de este último como un elemento inserto en un sistema literario más general cuyos límites y conformaciones se sustentan en el citado horizonte de expectativas y en los cambios históricos, en tanto que institución, nos sitúa ante elementos que atañen a la pragmática y a la manera en que en la sociedad se construyen y edifican los edificios de tales instituciones. El hecho es que todo texto es leído sobre el trasfondo de una tradición y en el marco de unas convenciones y un momento histórico concreto que determinan la manera -apropiada o desviada- en que dicho texto es recibido y comprendido. En suma, es lo que se suele entender como "competencia textual", aunque, como aduce Pozuelo Yvancos, el adjetivo textual no haga aquí justicia al fuerte componente pragmático del concepto ${ }^{10}$ y quizás sea preferible hablar de códigos y subcódigos, a la manera de Umberto Eco, quien, en Lector in fabula, habla de códigos y subcódigos y define el complejo que éstos fabrican como "competencia enciclopédica" construida sobre la base de elementos entre los que se encuentra lo que denomina hipercodificación retórica y estilística, entre cuyas reglas de hipercodificación se hallan a su vez las reglas de género ${ }^{11}$. Tal codificación remite, en la línea de lo expuesto hasta el momento, al aparente oxímoron de una tradición cambiante, continuamente reelaborada por las fuerzas sociales y culturales en juego.

Un elemento capital que interviene en la codificación de esta tradición es precisamente la reflexión de los estudiosos y los propios creadores. Si, como apunta Todorov, convenimos "en llamar géneros únicamente a las clases de textos que han sido percibidas como tales en el curso de la historia", resulta que "los testimonios de esa percepción se encuentran, ante todo, en el discurso sobre los géneros (discurso metadiscursivo) y, esporádicamente, en los propios textos" ${ }^{" 12}$. Como institución cultural, el género literario se construye de manera similar a otras instituciones culturales, cuya predicable esencialidad o universalidad, pese a su posible longevidad, son convenciones edificadas sobre el consenso y un proceso de elaboración y reelaboración en el que, forzosamente y en este caso, han de intervenir destacadamente quienes aportan el discurso metatextual.

En este sentido, Jean-Marie Schaeffer ha defendido lúcidamente la distinción, en el seno de la cuestión genérica, entre el género como categoría de clasificación retrospectiva y la genericidad en tanto que función textual. En el primer caso estaríamos hablando de un modo de lectura por el que en una proyección hacia atrás en el tiempo (Schaeffer habla de projection rétrospective) se postula una serie genérica; en el segundo caso, la genericidad habría de entenderse como "un factor productivo de la constitución de la textualidad" "13, y que Schaeffer identifica explícitamente con la architextualidad de Genette, que éste define como "el conjunto de categorías generales, o trascendentes -tipos de discursos, modos de enunciación, géneros literarios, etc.- del que surge cada texto individual"14 e incluye dentro

\footnotetext{
${ }^{10}$ Pozuelo Yvancos 1993: 221.

11 Eco 1981: 109 y ss. Los otros elementos son un diccionario básico, unas reglas de correferencia, unas selecciones contextuales, unas operaciones de inferencias y una hipercodificación ideológica.

${ }^{12}$ Todorov 1988: 36 .

${ }^{13}$ Schaeffer 1986: 199.

${ }^{14}$ Genette 1982: 7.
} 
de la categoría mayor de transtextualidad ${ }^{15}$. El concepto de genericidad parece más abstracto e inasible, pero desde la perspectiva de Schaeffer viene a entenderse como la función productiva consistente en que un texto en trance de elaboración utilice como material un modelo genérico previo, sin llegar a reduplicarlo, en principio.

De este modo, genericidad (como función textual) y género (como clasificación retrospectiva) son conceptos diferenciados, aunque relacionados en la medida en que son facetas de un mismo fenómeno. Schaeffer explica gráficamente esta cuestión del modo siguiente: una serie textual $\{a, b, c, d, e, f, g\}$, denominada género $G$, es establecida como tal sobre la base de una clasificación retrospectiva fijada cuando el último texto ha entrado en el circuito de la comunicación social. Pero para examinar la relación de, por ejemplo, $c$ con su género habría que tomar, más bien, en consideración una subserie $G_{l}$ (y no $G$ ) que se correspondería con el modelo genérico en el momento de su aparición; de ese modo, la perspectiva tomada atendería a la genericidad; sin embargo, interrogarse sobre la relación de $c$ con $G$, atendería más bien a la clasificación retrospectiva ${ }^{16}$.

Así pues, tendríamos dos formas, diferentes y necesariamente diferenciables, pero complementarias, de dar cuenta del género. A lo largo de la historia de la crítica y de la Teoría de la Literatura se ha puesto de manifiesto la ya aludida importancia del discurso teórico sobre la propia literatura -es decir, de la práctica crítica, del discurso meta-literarioy la delimitación de los géneros, que se erigen así en fruto de la convención institucional cultural, al ser precisamente tarea de los estudiosos reflexionar sobre la realidad histórica del momento y del pasado. Se percibe también la existencia de una posición equilibrada que intenta casar las diferentes tendencias respecto a los géneros y que modernamente pasa a distinguir entre géneros históricos, cambiantes y convencionales, y modos de presentación, universales, estables y dependientes no tanto de la reflexión y la convención histórica como de la propia economía expositiva de la obra literaria, como el modo de enunciación ${ }^{17}$.

Por tanto, se alza como elemento determinante la existencia de una teoría que sustente la reflexión de la que emana tal o cual clasificación retrospectiva. En este sentido, el cambio, el desarrollo o la evolución de la teoría puede implicar a su vez cambios en los límites de la clasificación al quedar los criterios de ésta alterados. En este sentido, Rollin ha afirmado que "la clasificación de los géneros sólo se da cuando viene guiada por una teoría, teoría que quede por encima de la escisión natural-convencional. (...) Sin una teoría, la clasificación literaria es algo análogo, en el mejor de los casos, a la historia natural en biología, el reconocimiento al azar de semejanzas entre particulares"18.

\footnotetext{
${ }^{15}$ La transtextualidad es definida por Genette (1982: 7) como "la trascendencia textual del texto (...) todo lo que lo pone en relación, manifiesta o secretamente, con otros textos".

${ }^{16}$ Véase Schaeffer 1986: 198.

${ }^{17}$ Para un panorama detallado de la cuestión genérica en la Teoría de la Literatura, consúltese García Berrio \& Huerta Calvo 1999; García Berrio 1994: 575 y ss, y Genette 1986.

${ }^{18}$ Rollin 1988: 149.Tras este pasaje, Rollin sigue: "Hasta cierto punto, por supuesto, la teoría clásica del género estaba basada en una teoría que no estaba bien articulada y que no resultaba adecuada para examinar los procesos literarios reales, esto es, las formar literarias enumeradas por Aristóteles...". Relaciónese esto con las reflexiones de Genette 1986.
} 


\section{EL CASO MARROQUí}

El ámbito marroquí ofrece un ejemplo diáfano de transformación del canon novelístico y los límites del género a partir puramente de la reflexión teórica de los estudiosos y los propios creadores y el proceso apuntado de formación retrospectiva. Por una parte, se trata de un género que en el ámbito marroquí es perfectamente acotable en el tiempo en lo que concierne a su comienzo, y donde las oscilaciones en estos límites se deben precisamente a los cambios de criterios teóricos; y ello debido a la tardía aparición del género -años cincuenta o sesenta según los listados bibliográficos- y a la exigua producción, que no despega hasta los años ochenta.

En efecto, esta progresión productiva desde una insólita penuria de la producción hasta un relativo florecimiento se ve en un simple vistazo al libro de 'Abd al-Rahīm al-'Allām (2000) titulado al-Riwāya al-magribiyya bi-l-luga al-'arabiyya (La novela marroquí en lengua árabe) y editado por la Asociación de Escritores de Marruecos (AEM). Pese a que esta obra recoge junto a las novelas otros textos clasificables dentro del ámbito autobiográfico -como se reconoce en el propio libro-, lo cierto es que es igualmente válida para captar el incremento de la producción editorial de textos narrativos y goza, además, de la autoridad que supone ser editado por la AEM. Así, un rápido recuento del listado, que se inicia en 1942 y se extiende hasta 1999, arroja el siguiente balance: en 1980 habían sido publicadas 40 obras, cuatro de ellas ese mismo año (aunque algunos de estos textos son más bien autobiografías), pero en 1986 el número se había duplicado ya hasta las 80 obras, y diez años más tarde, en 1996, el número se había vuelto a duplicar hasta superar los 160 textos.

Por otra parte, el proceso de emergencia y de progresiva acumulación de obras del género novelístico en Marruecos ha venido acompañado $\mathrm{y}$, en ocasiones, aparentemente tutelado por una producción teórica y crítica paralela, en lo que supone un ejemplo claro y diáfano de la interrelación entre producción artística y elaboración teórico-crítica con el fin de configurar un canon genérico. Es, además, un ejemplo de las alusiones de las páginas precedentes a los intentos de la crítica por casar sus elaboraciones teóricas con la realidad histórica y cambiante de los textos. Esta cuestión se relaciona también, sobre todo en los primeros momentos del género novelístico, con la cuestión de la configuración, en ocasiones laboriosa, de un tipo de discurso acorde con las necesidades de un género aún en ciernes y del que en sus primeros momentos, y aún más adelante, no se conocen sus rasgos ni sus límites todavía por cristalizar.

Sabri Hafez estudia en su obra The Genesis of Arabic Narrative Discourse (1993) la emergencia de ese nuevo discurso narrativo literario como el resultado de una mezcla de elementos coadyuvantes: la extensión de la educación, un cierto nivel de emancipación de la mujer, la aparición del nacionalismo, la expansión del periodismo y la generalización y popularización de las traducciones ${ }^{19}$. Las reflexiones de Hafez, centradas más bien en los casos egipcios y libaneses, son, mutatis mutandis, perfectamente extrapolables al caso marroquí, que, por su parte, ha sido exhaustivamente estudiado en sendas investigaciones

\footnotetext{
${ }^{19}$ Hafez 1993: 97, y capítulos 1-4.
} 
llevadas a cabo por Fātima Azrūwīl (1989) y Gonzalo Fernández Parrilla (2000) ${ }^{20}$. En su trabajo Azrūwīl delimita un recorrido unísono y paralelo entre los conceptos de novela que la crítica en Marruecos manejaba desde los años cincuenta hasta finales de los ochenta, y la marcha de la producción novelística propiamente dicha. Tal recorrido pasa por tres fases que, sucesivamente, podrían ser denominadas como fase de fundación, fase sociológica y fase artística, y que van desde mediados de los años sesenta hasta principios de los ochenta. En esto Azrūwīl es coincidente con la generalidad de la crítica marroquí.

Característica importante del caso marroquí es que uno de los ejes de su discurso teórico-crítico sobre la novela se sitúa desde los sesenta en torno a los límites del género y, más concretamente, a la cuestión de la obra inaugural. La piedra clave que soporta la cuestión del nacimiento del género es la del propio concepto de novela. A esto se debe que entre los años sesenta y los ochenta el cetro de novela inaugural pasara de una obra a otra de acuerdo con los flujos y reflujos de las sucesivas corrientes y tendencias teóricas. Así, en una primera etapa se vivió un largo consenso, en este sentido, en torno a Fì l-tufüla (De la niñez, 1957 ${ }^{21}$ ), de 'Abd al-Mayīd bn. Yallūn, que duró toda la década de los sesenta y setenta y que se debió a que la crítica, en primer lugar, obvió el hecho teórico crucial de que se trataba claramente de una autobiografía (lo que dice mucho de la confusión conceptual imperante $)^{22}$; en segundo lugar, a que esa misma crítica se dejó llevar durante los sesenta por la euforia nacionalista (la obra muestra intensos ramalazos patrióticos y aparece como libro en 1956, precisamente en las mismas fechas de la independencia de Marruecos), y, más tarde en los setenta, por una cierta inercia favorecida por el desprecio de la crítica -en esta década predominantemente social-realista y que Azrūwīl denomina, como hemos apuntado, "sociológica"- hacia una obra manifiestamente nacionalista como Dafannā almād (Hemos enterrado el pasado, 1967), de 'Abd al-Karīm Gallāb ${ }^{23}$, aparecida a finales de la década anterior, pero destinada en los ochenta a erigirse en novela fundacional.

Con la llegada de los años ochenta se matiza el concepto social-realista y aparece un concepto artístico de la novela. Este concepto por el cual, como explica Azrūwīl:

“...el crítico no se limita a lanzar juicios de valor tanto como a descifrar la obra literaria, afirmando la libertad del novelista para tratar con la realidad y elaborar ésta artísticamente.

\footnotetext{
20 Se trata de la tesis doctoral de Fernández Parrilla, editada en microfichas: La literatura marroqui contemporánea. La novela y la crítica literaria, Cuenca, Servicio de Publicaciones de la Universidad de CastillaLa Mancha. Este estudio se encuentra actualmente en prensa.

${ }^{21}$ Esta novela ha sido traducida al castellano por Salvador Peña: De la niñez, Ediciones del Oriente y del Mediterráneo, Madrid, 1999.

22 "La evolución de este concepto revela una contradicción, que surge cuando observamos la coincidencia de los críticos, desde que la crítica novelística echara a andar, en considerar una autobiografía ( F $\bar{l}$ l-tufūla) como el punto de partida de la novela en Marruecos, sin mostrar ninguna reserva o precaución al proclamar esta postura" (Azrūwīl 1989: 101).

${ }^{23}$ Como muestra, valga el parecer de Idrīs al-Nāqūrī, uno de los críticos más destacados de la época: “...sin embargo, Gallāb insiste en darnos una novela de corte clásico y de pobre calidad. (...) Gallāb fue en efecto consecuente consigo mismo y fiel a sus fundamentos materiales cuando se limitó a ser vehículo de expresión de su clase social y a asumir la defensa de ésta con empeño y valentía. (...) Hemos enterrado el pasado no es, por ejemplo, sino uno de tales instrumentos con los que se arma para ayudar a los planes del político" (Idris al-Nāqūrī $1985^{3}$ : 66).
} 
(...) los críticos rehuyen poner un límite definitivo a la novela y reconocen la multiplicidad de tipos novelísticos y su variedad de acuerdo con las sociedades y las épocas"24.

Esto supone, de hecho, abrir las puertas a un cierto eclecticismo que depende, de nuevo, de los posicionamientos teóricos particulares de cada crítico. Así, en 1985 Ahmad alMadīn̄i afirmaba que Dafannā al-mād̄ "da cuerpo a las características completas de la novela de Marruecos" ${ }^{\prime 25}$; pero un año antes, Ahmad al-Yābūrī defendía el carácter novelístico de obras como al-Zāwiya (La zagüía, 1942) y Wazìr Garnāta (El ministro de Granada, 1950), obras que, desde la autobiografía y el relato biográfico histórico novelado respectivamente, se situaban en el límite del género novelístico en Marruecos. La postura de al-Yābūrī introducía un elemento nuevo en la discusión crítica en un contexto de amplia repercusión como los actos del Congreso sobre la Novela Marroquí celebrado en 1984 en Rabat.

Estos vaivenes respecto a la obra inaugural abundan en el argumento de la importancia decisiva de la reflexión metaliteraria para la delimitación del propio objeto de estudio. En este sentido, la cuestión del "umbral del género", o punto de partida, tiene que ver con el concepto de novela $\mathrm{y}$, por tanto, con la capacidad para marcar los límites con géneros adyacentes.

'Abd al-Rahīm al-'Allām, al principio de su opúsculo sobre la novela marroquí, considera de importancia relativa el problema del principio del género novelístico en Marruecos y el papel de la autobiografía, así como la posibilidad de que la novela en Marruecos, al contrario que en Europa, provenga de la autobiografía ${ }^{26}$. La cuestión no carece de importancia, pero el empeño en buscar y localizar la obra primera, la referencia sobre la que se construye por acumulación el género, puede llevar a errores si no se tiene en cuenta el propio concepto variable de género. En la medida en que hemos considerado el género literario una institución cultural, y por tanto histórica, la obra primera se someterá también a los vaivenes que sufra la institución en la que se incluya. A esto es a lo que se refiere al-'Allām cuando habla de los vaivenes del consenso sobre el primer texto novelístico de Marruecos entre Fì l-tufüla (1956), Dafannā al-mādī (1966), al-Gurba (El exilio, [1971]) y al-Zāwiya (1942).

Partiendo de la distinción entre novela y autobiografía y sin mezclar géneros, parece existir actualmente cierto consenso de la crítica en que la primera novela marroquí moderna es Dafannā al-mādī27. Pues, aunque se acepte la importancia de Fì l-tufūla, la adscripción de ésta al marco de la autobiografía ${ }^{28}$ deja la novela de "Abd al-Karīm Gallāb como primer exponente del género. Así, Ahmad al-Madīnī concederá que Fì l-tufūla constituye "el principio artístico de la novela marroquí", aunque "lo cierto es que $F \bar{l} l$-tufūla de "Abd alMayīd bn. Yallūn se inserta en el género de la autobiografía". La tesis de al-Madīn̄i es que la autobiografía es el molde artístico de la pulsión narrativa que anticipaba la novela, pero

\footnotetext{
${ }^{24}$ Azruwil 1986: 54.

${ }^{25}$ Al-Madini 1985: 54.

${ }^{26}$ Al-'Allam 2000: 6.

${ }^{27}$ Fernández Parrilla (2000) realiza un seguimiento detallado de estos vaivenes. Véanse especialmente los capítulos 7 - "La construcción del canon"- y 8 - "La reinvención del canon"-.

${ }^{28}$ Véase, sobre este punto, el trabajo sobre la autobiografía en Marruecos de ‘Abd al-Qādir al-Šāwī (2000).
} 
que es Dafannā al-mād $\bar{l}$, como hemos señalado más arriba, el texto que encarna en su opinión las características totales de la novela de Marruecos ${ }^{29}$. De alguna manera, la posición de al-Madīnī es un intento de casar la importancia concedida a $F \bar{l}$ l-tufūla con el hecho de que, en puridad, no es un relato de ficción ${ }^{30}$.

Muhammad Barrāda, en un texto crítico muy anterior ${ }^{31}$, ya apuntaba una postura similar a la de al-Madīnī. El primer texto que estudia Barrāda es $F \bar{l} l$-tufüla, y comienza intentando explicar el hecho de que el primer texto novelístico fuera una autobiografía ${ }^{32}$. El segundo texto examinado es el ya mencionado Dafannā al-mād $\bar{\imath}$, y el tercero es Y̌̃l al-zam' ( $L a$ generación de la sed, 1967), de Muhammad 'Azīz al-Habbābī, para pasar en el último apartado a repasar una serie de novelas que denomina "fracasadas" (al-riwāyāt al-fāšila), a saber: Amtār al-rahma (Las lluvias de la compasión, 1965), de 'Abd al-Rahmān al-Marīn̄i; Gadan tatabaddal al-ard (Mañana cambiará la tierra, 1967), de Fātima al-Rāwā; Būtaqat al-hayāh (El crisol de la vida, 1966), de al-Bakrī Ahmad al-Sibā‘ī; e Inna-hā al-hayāh (He aquí la vida, s. d., pero probablemente de mediados de los sesenta), de Muhammad alBū'nānī. Las razones de Barrāda para este duro calificativo son de índole formal: "penuria técnica" (al-iqdā' al-fann $\vec{\imath}$ y carencias en la "asimilación del desarrollo de la novela árabe" 33 . Estas novelas "fracasadas" se publican en el periodo que media entre la aparición de Fì l-tufüla (1956) y Dafannā al-mādì (1966). El mensaje que, en el fondo, parece querer expresar Barrāda -o al menos se colige de este planteamiento de las cosas- es que, al igual que opinaría al-Madini varios años más tarde, en 1985, Dafannā al-māà es la primera novela marroquí lo suficiente madura como para generar epígonos que den forma a un género.

\section{FINAL}

El propio concepto de género como institución cultural, con sus componentes sociales e históricos, requiere una cierta perspectiva para decidir qué texto es el que marca el referente; porque en este caso no se trata de dilucidar el primer texto, sino la referencia. Si Fì l-tufüla, con sus cualidades artísticas, es una autobiografía, y las novelas fracasadas no pueden constituirse en modelo ${ }^{34}$, parece que Dafannā al-mād $\bar{\imath}$ se yergue como el texto que inaugura institucionalmente el género en Marruecos. Cuando 'Abd al-Hamīd 'Aqqār afirma, en un texto más moderno (del año 2000), "Dafannā al-mādì puede ser considerada

\footnotetext{
${ }^{29}$ Al-Madīn̄i 1985: 42-44.

${ }^{30}$ Sobre esta cuestión comenta Roger Allen (1992: 205): “En Marruecos, por ejemplo, ‘Abd al-Majīd ibn Jallūn (n. 1919) publicó su novela $F \bar{l}$ l-tufüla ("De la niñez") en 1957. La clara tendencia hacia la autobiografía es mucho más evidente aquí que en obras anteriores; de hecho, es tal la atención hacia las descripciones detalladas de la vida en la ciudad inglesa de Manchester que, a pesar de los esfuerzos de Ibn Jallūn por conceder a su novela algún grado de ficcionalidad, parece más cercano a un relato de viajes, del tipo de al-Tahtawi Tajlīs al-ībrīz (...)".

${ }^{31}$ Barrāda 1969: 141-149.

${ }^{32}$ Azrūwīl (1989: 100) alude a esta cuestión: "Esta afirmación no llevó a los críticos a estudiar la relación entre estos dos géneros literarios [novela y autobiografía] como elementos diferenciados cuyos componentes podrían cruzarse y la naturaleza de estos componentes, en la medida en que les llevó más bien a estudiar las justificaciones -exteriores- que explicarían, en su opinión, que los creadores se vieran dirigidos a escribir sus autobiografías en una etapa histórica concreta".

${ }^{33}$ Barrāda 1969: 147.

${ }^{34}$ Barrāda 1969: 148.
} 
la primera novela, en el sentido europeo, publicada en Marruecos"35, expresa muy bien la idea de que lo importante no es el primer texto, sino el que marca la pauta a seguir. Como dice Claudio Guillén, "sólo el tiempo histórico puede demostrarnos que un modelo ha llegado efectivamente a erigirse en género" ${ }^{36}$. Así, los precedentes de Dafannā al-mād̄̄, o no eran ficción o no fueron modelos aceptables, como bien ha sabido ver el crítico egipcio Yābir 'Asfūr en un pasaje en el que resume con precisión el caso marroquí que hemos abordado aquí:

"Los propios estudiosos marroquíes delimitan a mediados de los sesenta el punto de partida real de la aparición efectiva del género literario de la novela en Marruecos, cuando se sucedieron novelas significativas de escritores marroquíes que intentaron poner los fundamentos de este género de una manera efectiva, en comparación con los intentos precedentes, que fueron incapaces de imponer su presencia o de dejar huella"37.

\section{REFERENCIAS BIBLIOGRÁFICAS}

AL-'ALLĀM, 'Abd al-Rahīm (2000): Al-riwāya al-magribiyya bi-l-'arabiyya. Mina lta'sīs ilà l-imtidād. Bīblīyūgrāfiyya 1942-1999 [La novela marroquí en árabe. De la fundación a las extensiones. Bibliografía 1942-1999]. Manšūrāt Ittihād Kuttāb al-Magrib [Publicaciones de la Asociación de Escritores Marroquíes].

ALLEN, Roger (1992): “The Mature Arabic Novel Outside Egypt”, en Muhammad Badawi (ed.), Modern Arabic Literature. The Cambridge History of Arabic Literature, Cambridge. Cambridge UP, pp. 193-222.

AL-MADĪNĪ, Ahmad (1985): Fì l-adab al-magribī al-mu'āsir [Sobre la literatura marroquí contemporánea]. Casablanca. Dār al-Našr al-Magribiyya.

AL-NAQŪRĪ, Idrīs (1985³): Al-mustalah al-muštarak. Dirāsāt fì l-adab al-magribī almu'āsir [El paradigma común. Estudios sobre literatura marroquí contemporánea]. Casablanca. Dār al-Našr al-Magribiyya [1977].

AL-YĀBŪRĪ, Ahmad (1984): “Takawwun al-jitāb al-riwā'̄o. Al-riwāya al-magribiyya namūdiyan" ["La formación del discurso novelístico. La novela marroquí como ejemplo"], $\bar{A} f a \bar{q}, \mathrm{n}^{\circ} 3-4,13-19$.

'AQQĀR, 'Abd al-Hamīd (2000): Al-riwāya al-magribiyya. Tahawwulāt al-luga wa-l-jitāb [La novela magrebi. Las transformaciones de la lengua y del discurso]. Casablanca. Širkat al-Našr wa-1-Tawzī'.

‘ASFŪR, Gābir (1999): Zaman al-riwāya [El tiempo de la novela]. El Cairo. Al-Hay'a alMisriyya al-'Āmma li-1-Kitāb.

\footnotetext{
35 'Aqqār 2000: 24.

${ }^{36}$ Guillén 1985: 150.

37 'Asfür 1999: 319-320.
} 
AZRŪWĪL, Fātima al-Zahrā' (1989): Mafāhīm naqd al-riwāya bi-l-Magrib. Masādiru-hā al-'arabiyya wa-l-aynabiyya $[$ Los conceptos de la crítica novelística en Marruecos. Sus fuentes árabes y extranjeras]. Casablanca. Fenek.

BARRĀDA, Muhammad (1969): "Al-usus al-nazariyya li-l-riwāya al-magribiyya almaktūba bi-1-"arabiyya" ["Los fundamentos teóricos de la novela marroquí escrita en árabe"] (publicado como anexo en "Abd al-Kabīr al-Jatībī (1971), alRiwāya al-magribiyya [La novela marroqui]. Rabat. Manšūrāt al-Markaz alYāmi‘̄i li-1-Baht al-'Ilmī [Publicaciones del Centro Universitario para la Investigación Científica]).

ECO, Humberto (1981): Lector in fabula. Barcelona. Lumen.

FERNÁNDEZ PARRILLA, Gonzalo (2000): La novela en Marruecos. Un nuevo género literario en el proceso de formación de una literatura árabe nacional: el papel de la crítica. Servicio de Publicaciones de la Universidad Autónoma de Madrid (microfichas).

GARCÍA BERRIO, Antonio (1994): Teoría de la Literatura (La construcción del significado poético). Madrid. Cátedra.

GARCÍA BERRIO, Antonio \& HUERTA CALVO, Javier (1999): Los géneros literarios: sistema e historia. Madrid. Gredos.

GENETTE, Gérard (1986): "Introduction à l'architexte", en AA. VV., Théorie des genres, 89-159. París. Seuil (Primera aparición Poétique, 1979).

GENETTE, Gérard (1982): Palimpsestes. París. Seuil.

GUILLÉN, Claudio (1985): Entre lo uno y lo diverso. Introducción a la Literatura Comparada. Barcelona. Crítica.

HAFEZ, Sabri (1993): The Genesis of Arabic Narrative Discourse. Londres. Saqi Books.

JAUSS, Hans Robert (1986): "Littérature médiévale et théorie des genres", en AA. VV., Théorie des genres, 37-76. París. Seuil (Primera aparición en AA.VV. 1970: Grundriss der romanischen Literaturen des Mittelalters. Heidelberg).

POZUELO YVANCOS, José María (1993): Poética de la ficción. Madrid. Síntesis.

ROLLIN, Bernard (1988): "Naturaleza, convención y teoría del género", en Manuel Garrido Gallardo (ed.), Teoría de los géneros literarios, 129-153. Madrid, Arco/Libros S.A. (original en inglés en Poetics 10 (1981) 127-143).

AL-ŠĀWİ, 'Abd al-Qādir (2000): Al-kitāba wa-l-wuyūd. Al-sīra al-d̄ātiya fì l-Magrib [La escritura y la existencia. La autobiografía en Marruecos]. Casablanca. Afrīqiyaal-Šarq.

SCHAEFFER, Jean-Marie (1986): "Du texte au genre", en AA. VV., Théorie des genres, 179-205. París. Seuil. (Artículo aparecido originalmente en Poétique, 53 [1983].)

TODOROV, Tzvetan (1988): "El origen de los géneros", en Manuel Garrido Gallardo (ed.), Teoría de los géneros literarios. Madrid. Arco/Libros S.A. (Nueva versión de “The origin of Genres". New Literary History. VIII, 1 (1976) 145-158).

WELLEK, René \& WARREN, Austin $\left(1966^{4}\right)$ : Teoría literaria. Madrid. Gredos [1948]. 